From (3), $R_{q(y) \cdot z}$ is onto and $L_{t(y)}$ is $1-1$. Since $G$ is finite, both are bijections. In particular, letting $a$ be any element of $G$, there is an element $b$ such that $t(a) \cdot b=t(a)$. Thus $b=((t(a)) b)(q(a) \cdot z)$ $=(t(a))(q(a) \cdot z)$. Since $L_{t(a)}$ is $1-1, q(a) \cdot z$ is independent of $z$. Thus $L_{q(a)}$ is constant.

Let $c=q(a) \cdot z$, independent of $z$. Replacing $z$ by $q(y) \cdot z$ gives $c=q(a)(q(y) \cdot z)$. From (2), with $x$ replaced by $c$,

$$
c=(t(y) \cdot c)(q(y) \cdot z) \text {. }
$$

Since $R_{q(y) \cdot z}$ is $1-1, t(y) \cdot c=q(a)$, which is independent of $y$. Thus $t(y) \cdot c=t(a) \cdot c$ and since $R_{c}$ is $1-1, t(y)=t(a)$.

Now let $y$ be replaced by $d=t(a)$. This gives $t(d)=t(a)=d$. Thus $q(d)=d d \cdot t(d)=d d \cdot d=t(d)$.

Since $q(d)=t(d), L_{q(d)}$, which is constant, is equal to $L_{t(d)}$, which is a bijection. Thus $G$ has only one element.

\title{
REFERENCE
}

1. S. K. Stein, Finite models of identities, Proc. Amer. Math. Soc. 14 (1963), 216-222.

Training College, Huld, Yorkshire, England

\section{AN ELEMENTARY INTERPOLATION THEOREM}

\section{DAVID G. CANTOR AND R. R. PHELPS}

In this note we prove a simple lemma concerning interpolation properties of certain families of maps in semigroups. The main application is to the family of finite Blaschke products, considered as maps of the circle into itself. (Other applications are possible, but most of them are well known.)

Consider a semigroup $S$ and a family $F$ of maps from $S$ into $S$. We will call $F n$-transitive if for any $n$ distinct elements $\alpha_{1}, \alpha_{2}, \cdots, \alpha_{n}$ of $S$ and arbitrary elements $\beta_{1}, \beta_{2}, \cdots, \beta_{n}$ in $S$, there exists $f$ in $F$ such that $f\left(\alpha_{i}\right)=\beta_{i}, i=1,2, \cdots, n$.

Lemma. Suppose that $S$ is a semigroup with (two-sided) identity 1 and suppose there is an element $\beta$ in $S$ with $\beta \neq \beta^{2} \neq 1$. Assume, furthermore, that $F$ is a family of maps (from $S$ into $S$ ) which is closed under

Received by the editors December 17, 1963. 
the operations of composition and pointwise multiplication. Then $F$ is n-transitive provided it is 3-transitive.

Proof. It clearly suffices to show that given distinct elements $\alpha_{1}, \cdots, \alpha_{n}$ and arbitrary elements $\beta_{1}, \cdots, \beta_{n}$, there exist $n$ functions $f_{k}$ in $F$ such that $f_{k}\left(\alpha_{i}\right)=1(k \neq i)$ and $f_{k}\left(\alpha_{k}\right)=\beta_{k}$; we obtain the desired function by taking the product of the $f_{k}$. The proof of the existence of $f_{k}$ proceeds by induction, and to simplify the notation we assume that $k=1$. Suppose, then, that $n \geqq 4$ and that $F$ is $(n-1)$ transitive. The latter hypothesis enables us to choose $g$ in $F$ such that $g\left(\alpha_{1}\right)=\beta, g\left(\alpha_{i}\right)=1, i=2,3, \cdots, n-1$. Consider the element $\gamma=g\left(\alpha_{n}\right)$. If $\gamma \neq \beta$, we can choose $h$ in $F$ such that $h(\beta)=\beta_{1}, h(1)=1=h(\gamma)$ and hence we can let $f_{1}=h \circ g$. If $\gamma=\beta$, we choose $g_{1}$ such that $g_{1}\left(\alpha_{1}\right)=\beta$, $g_{1}\left(\alpha_{i}\right)=1, i=3,4, \cdots, n$ and this time we consider $g_{1}\left(\alpha_{2}\right)$. If this differs from $\beta$, we can obtain $f_{1}$ in the same manner as above. If $g_{1}\left(\alpha_{2}\right)=\beta$, let $g_{2}=g g_{1}$ and note that

$g_{2}\left(\alpha_{1}\right)=\beta^{2}, g_{2}\left(\alpha_{2}\right)=\beta=g_{2}\left(\alpha_{n}\right)$ and $g_{2}\left(\alpha_{i}\right)=1, i=3, \cdots, n-1$.

Since the elements $\beta, \beta^{2}$ and 1 are distinct, we can choose $h_{1}$ such that $h_{1}\left(\beta^{2}\right)=\beta_{1}, h_{1}(\beta)=1=h_{1}(1)$; the map $f_{1}=h_{1} \circ g_{2}$ has the desired properties and the proof is complete.

At the end of this note we will give an example which shows that this theorem may fail (even when $S$ is a finite commutative group) if every element of $S$ has order two. (To see that 2-transitivity need not imply 3-transitivity, let $S$ be the real numbers under addition and let $F$ be all linear functions on $S$.)

A finite Blaschke product is a complex function of the form

$$
f(z)=\lambda \prod_{k=1}^{m}\left(\frac{z-a_{k}}{1-\bar{a}_{k} z}\right)
$$

where $|\lambda|=1,\left|a_{k}\right|<1(k=1,2, \cdots, m)$ and $m \geqq 0$. (If $m=0, f$ is the constant function $\lambda$.) We do not assume that the $a_{k}$ are distinct. Clearly, the family of all finite Blaschke products is closed under multiplication, and it is well known that it is closed under composition. (This follows easily, for instance, from the fact that the nonconstant finite Blaschke products are precisely those functions which are analytic in (a neighborhood of) $|z| \leqq 1$ and which map the circle $|z|=1$ onto itself.)

THEOREM. If $\alpha_{1}, \alpha_{2}, \cdots, \alpha_{n}$ are distinct complex numbers of modulus one and if $\beta_{1}, \beta_{2}, \cdots, \beta_{n}$ have modulus one, then there exists a finite Blaschke product $f$ such that $f\left(\alpha_{i}\right)=\beta_{i}, i=1,2, \cdots, n$. 
Proof. We need only show that the finite Blaschke products are 3-transitive. We first show that they are 2-transitive. (It is obvious that the rotations $z \rightarrow \lambda z,|\lambda|=1$, yield 1 -transitivity.) Thus, suppose that $\alpha_{1}, \alpha_{2}, \beta_{1}$ and $\beta_{2}$ have unit modulus and that $\alpha_{1} \neq \alpha_{2}$. By a rotation, we can assume that $\alpha_{1}$ is in the upper half-plane, and that $\alpha_{2}=\bar{\alpha}_{1}$. (Simply multiply by $\lambda$, where $|\lambda|=1, \operatorname{Re} \lambda \alpha_{1}=\operatorname{Re} \lambda \alpha_{2}$ and $0<\operatorname{Im} \lambda \alpha_{1}=-\operatorname{Im} \lambda \alpha_{2}$.) Assume for the moment that $\beta_{1} \neq \beta_{2}$; then we can rotate these points in a similar manner, and hence we can suppose that $\beta_{1}$ is in the upper half-plane and $\beta_{2}=\bar{\beta}_{1}$. We then choose a real number $a$ such that $|a|<1$ and the function $f(z)=(z-a)(1-a z)^{-1}$ maps $\alpha_{1}$ onto $\beta_{1}$. (Explicitly, take $\left.a=\left(\beta_{1}-\alpha_{1}\right)\left(1-\alpha_{1} \beta_{1}\right)^{-1}\right)$. Since $a$ is real, $f(\bar{z})=[f(z)]-$, so $f\left(\alpha_{2}\right)=\beta_{2}$. The final function which does the job, then, consists of a rotation followed by the above $f$ which is followed by another rotation. Note that the resulting Blaschke product has only one nonconstant factor. Suppose, now, that $\beta_{1}=\beta_{2}$. (We could take a constant function in this case, but for later purposes we want to do better than this.) Again, by a rotation we can assume that $\beta_{1}=\beta_{2}=1$, and by what we have just proved there is a Blaschke product $f_{1}$ such that $f_{1}\left(\alpha_{1}\right)=1, f_{1}\left(\alpha_{2}\right)=-1$. The function $f=f_{1}^{2}$ has the desired properties; furthermore, $f$ has exactly two nonconstant factors, and hence is a two-to-one map. Consider, finally, the case $n=3$. As in the proof of the lemma, we only show that if $\alpha_{1}, \alpha_{2}, \alpha_{3}$ are distinct and $\beta_{1}, \beta_{2}, \beta_{3}$ are arbitrary, then there exists a finite Blaschke product which maps $\alpha_{1}$ and $\alpha_{2}$ onto 1 , and $\alpha_{3}$ onto $\beta_{3}$. To this end, choose a two-to-one map $f$ (as above) which takes $\alpha_{1}$ and $\alpha_{2}$ onto 1 ; it follows that $f\left(\alpha_{3}\right) \neq 1$. We then choose $h$ such that $h(1)=1$ and $h\left(f\left(\alpha_{3}\right)\right)=\beta_{3}$; the function $h$ of has the desired properties and the proof is complete.

Consider the following example: Let $S$ be the noncyclic group of order four (the "four group") and let $F$ consist of all maps of $S$ into $S$ which are either one-to-one, two-to-one or four-to-one. It is easy to check that $F$ is closed under composition, and that $F$ consists precisely of those maps $f$ for which $\prod_{\alpha \in S} f(\alpha)=1$. This latter property implies immediately that $F$ is closed under multiplication. It is clear that $F$ is 3 -transitive, but that no nonconstant map in $F$ can map three distinct elements of $S$ into the same element; it follows that $F$ is not 4-transitive.

UNIVERSITY OF WASHINGTON 